\title{
Severe neuropsychiatric systemic lupus erythematosus successfully treated with rituximab: an alternative to standard of care
}

This article was published in the following Dove Press journal:

Open Access Rheumatology: Research and Reviews

II September 2017

Number of times this article has been viewed

\section{Elisabetta Chessa \\ Matteo Piga \\ Alberto Floris \\ Alessandro Mathieu \\ Alberto Cauli}

Rheumatology Unit, University Clinic AOU of Cagliari, Cagliari, Italy
Correspondence: Matteo Piga Rheumatology and Rheumatology Unit, University Clinic AOU of Cagliari, ss 554 - CAP 09042, Monserrato, Cagliari, Italy

Tel +397051096381

Fax +397051096382 ext 513157

Email matteopiga@alice.it

\begin{abstract}
Demyelinating syndrome secondary to systemic lupus erythematosus (DS-SLE) is a rare encephalomyelitis burden with a high risk of disability and death. We report on a 49-year-old Caucasian woman with systemic lupus erythematosus (SLE) complicated by severe cognitive dysfunction, brainstem disease, cranial nerve palsies, weakness and numbness in limbs and multiple discrete magnetic resonance imaging (MRI) areas of damage within the white matter of semioval centers, temporal lobe, external capsule, claustrum, subinsular regions and midbrain. She also had multiple mononeuritis diagnosed through sensory and motor nerve conduction study. She was diagnosed with severe DS-SLE prominently involving the brain and was treated with $500 \mathrm{mg}$ methylprednisolone (PRE) pulses for 3 consecutive days, followed by one single pulse of $500 \mathrm{mg}$ cyclophosphamide, and $1 \mathrm{~g}$ rituximab, which was then repeated 14 days later. PRE $25 \mathrm{mg}$ /day, rapidly tapered to $7.5 \mathrm{mg}$ /day in 6 months, and mycophenolate mofetil $1 \mathrm{~g} /$ day were prescribed as maintenance therapy. She had progressive and sustained improvement in neurological symptoms with almost complete resolution of brain MRI lesions after 1 year. B-cell depleting therapy could be considered as a possible alternative to standard of care in the management of severe inflammatory neuropsychiatric SLE but it should be associated with a conventional immunosuppressant as maintenance treatment to reduce the risk of flare and reduce corticosteroids dose.
\end{abstract}

Keywords: systemic lupus erythematosus, neuropsychiatric lupus, rituximab, demyelinating syndrome, brain MRI

\section{Introduction}

Neuropsychiatric involvement in systemic lupus erythematosus (NPSLE), as defined by the American College of Rheumatology (ACR), occurs in 37-91\% of patients but only $18-33 \%$ of the events are attributable to systemic lupus erythematosus (SLE). ${ }^{1-3}$ NPSLE has a great impact on patients due to higher risk of hospitalization, impaired quality of life and death. ${ }^{4-6}$ Demyelinating syndrome secondary to systemic lupus erythematosus (DS-SLE) is an encephalomyelitis that may mimic other causes of primary and secondary demyelination as multiple sclerosis (MS), infections and malignancies. ${ }^{1-3}$ A recent survey estimated the prevalence of DS-SLE as $0.9-1.3 \%$ and the incidence rate as $1.2-1.5$ cases/1000 patient-years. ${ }^{7}$ Although rare, it is a life-threatening condition with great risk of disability and death related to a high relapsing rate. ${ }^{7}$

European League Against Rheumatism (EULAR) recommendations underscored the need for a two-hit therapeutic strategy in severe inflammatory NPSLE, with initial intravenous corticosteroids and cyclophosphamide (CYC; first hit) treatment aimed to control inflammation and prevent irreversible damage followed by immunosuppressive maintenance therapy (second hit) pointed to prevent relapse and spare steroids. ${ }^{8} \mathrm{~B}$-cell 
depleting therapy (BCDT) with rituximab (RTX) is successfully used as second-line treatment in refractory and relapsing NPSLE cases, but it has been considered as first-line treatment in SLE to reduce the overall steroid burden. ${ }^{8-10}$ Here, we take advantage of an illustrative case to briefly discuss the possible role of RTX as part of the two-hit treatment strategy for management of severe inflammatory NPSLE. Written informed consent has been provided by the patient to have the case details and any accompanying images published.

\section{Case report}

On February 2016, a 49-year-old Caucasian woman with SLE was referred to our Rheumatology Department for cognitive dysfunction, neurological disturbances and worsening of renal function. Fifteen years before she was diagnosed with SLE on the basis of polyarthritis, photosensitivity, malar rash, leukopenia, antinuclear antibodies positive and low C3 and C4 complement fractions. She also had secondary Sjogren's syndrome, hypertension, tobacco addiction and steroids-induced Cushing's syndrome. On June 2015, she was admitted to another hospital for acute vomiting, left facial nerve palsy and ataxia. She was diagnosed with NPSLE and basal ganglia calcification (Figure 1) and then treated with $1 \mathrm{~g}$ methylprednisolone (MPRE) pulses for 5 consecutive days followed by prednisolone (PRE) $60 \mathrm{mg} /$ daily and hydroxychloroquine $400 \mathrm{mg}$ /daily, with relapse during PRE dose reduction when reaching the $7.5 \mathrm{mg} /$ day dosage.

On admission to our department, her husband reported that she had a progressive worsening of neurological disturbances in the last 6 weeks. On neurological examination, she had severe cognitive dysfunction in simple and complex attention, reasoning, problem solving and memory, being even unable to recall her age and the exact date. Bilateral nystagmus, diplopia, dysarthria, lower-limb ataxia and bilateral sixth and left seventh cranial nerve palsies were found. She also had weakness in the right side of her body, lower limbs sensory loss and hyperreactive deep tendon reflexes with left foot dorsiflexion deficit. Meningeal signs were absent. Blood pressure was 120/70 mmHg. Alopecia and bilateral Jaccoud arthropathy were also present. Blood tests showed anemia (hemoglobin $8.4 \mathrm{~g} / \mathrm{dL}$ ) with no hemolysis, erythrocyte sedimentation rate $81 \mathrm{~mm} / \mathrm{h}$, C-reactive-protein $38 \mathrm{mg} / \mathrm{dL}$ (normal range $0-1 \mathrm{mg} / \mathrm{dL}$ ), antinuclear antibodies 1:160 homogenous pattern, anti-dsDNA $2.4 \mathrm{UI} / \mathrm{L}$ ( $<7 \mathrm{UI} / \mathrm{L}$ by Farr), C3 $72 \mathrm{mg} / \mathrm{dL}$ (90-180 mg/dL), C4 $11 \mathrm{mg} / \mathrm{dL}(10-40 \mathrm{mg} / \mathrm{dL})$ and anti-Ro/SSA positive. Serum creatinine was $2 \mathrm{mg} / \mathrm{dL}$, and glomerular filtration rate was $31 \mathrm{~mL} / \mathrm{min}$ with proteinuria of $0.6 \mathrm{~g} /$ day but no active urinary sediment. Blood glucose, liver function tests, electrolytes, copper, ceruloplasmin, B12 vitamin and folate serum levels were within normal range. Serum anti-aquaporin-4, anti-glutamic acid decarboxylase, anti- $N$-methyl-D-aspartate receptor, anti- $\gamma$-aminobutyric acid receptor, anti-voltage-gated potassium channels and anti-AMPA receptor autoantibodies were negative. Cerebral spinal fluid (CSF) analysis revealed increased protein content $(46.8 \mathrm{mg} / \mathrm{dL})$ and pleocytosis (10 lymphocytes) with neither intrathecal $\operatorname{IgG}$ synthesis nor oligoclonal bands. Blood and CSF microbiological stains and cultures for bacteria, fungi and mycobacteria were negative. Blood and CSF search for CMV, VZV, HSV and JCV DNA as well as Borrelia burgdorferi, Treponema pallidum, Rickettsia and Brucella serological tests were negative. Screening for malignancies was negative. Electroencephalogram and visual and somatosensory evoked potentials were normal. Electromyography revealed left common peroneal nerve and bilateral sural nerves motor and sensory conduction abnormalities, diagnosed as multiple mononeuritis. Brain magnetic resonance imaging (MRI) confirmed basal ganglia calcifications and showed a dramatic

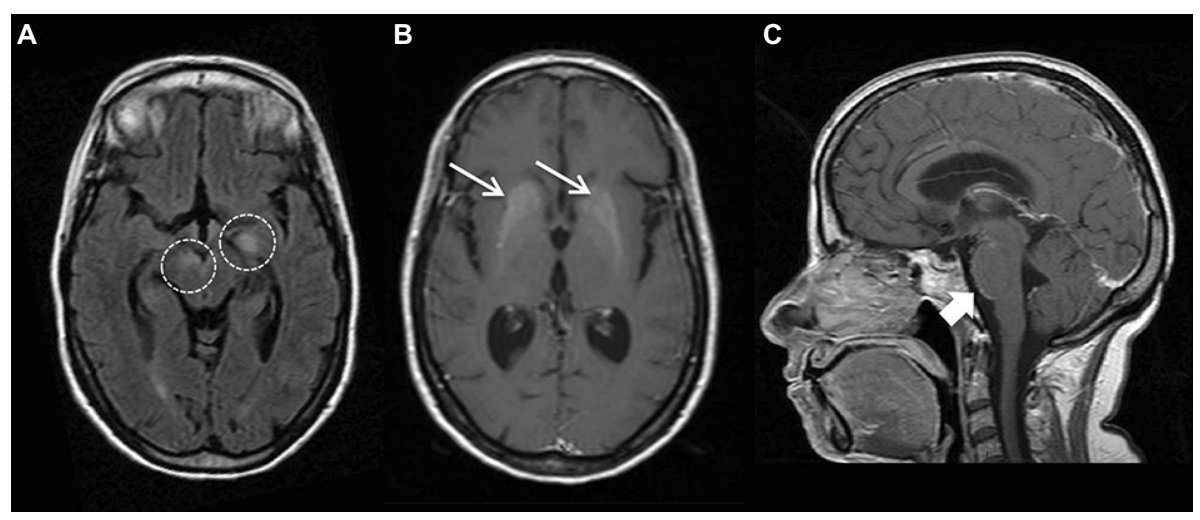

Figure I MRI findings at onset of neurological disturbances.

Notes: Brain MRI images from June 2015 showed (A) T2 hyperintense lesions in left temporal lobe and brainstem (circles), (B) TI hyperintense lesions in basal ganglia (thin arrows), known as calcifications, and (C) leptomeningeal contrast enhancement at midbrain-pons junction (arrow).

Abbreviation: MRI, magnetic resonance imaging. 


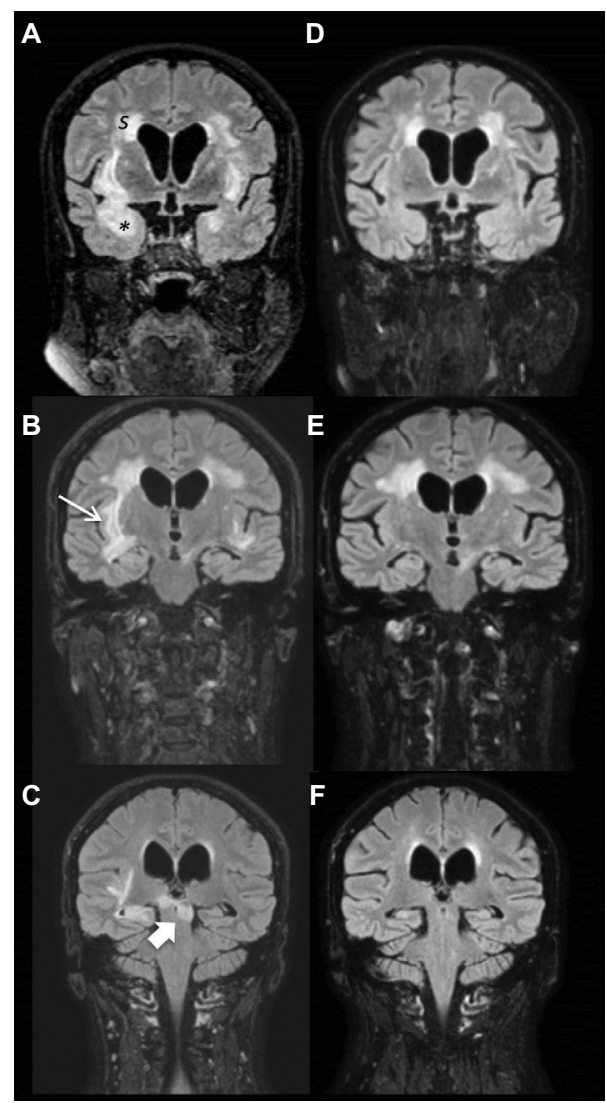

Figure 2 Brain MRI images before and after B-cell depleting therapy. Notes: Brain MRI images from February 2016 showed extensive and bilateral focal or confluents subcortical and deep WM lesions, hyperintense in long TR sequences, involving (A) semioval centers $(S)$, temporal lobe $(*),(B)$ external capsule, claustrum and subinsular regions (thin arrow) and (C) midbrain (arrow) without contrast enhancement. Moderate subcortical atrophy with dilation of lateral ventricles. Brain MRI in September 2016, 6 months after RTX treatment, showed reductions in numbers and size of hyperintensity lesions in WM, especially in temporal lobes bilaterally ( $\mathbf{D}$ and $\mathbf{E})$ and brainstem $(\mathbf{F})$.

Abbreviations: MRI, magnetic resonance imaging; WM, white matter; RTX, rituximab.

increase in the number and size of previous white matter (WM) lesions (Figure 2) with hyperintensity on diffusionweighted images and enhanced apparent diffusion coefficient suggesting vasogenic edema (Figure 3).

She was diagnosed with active SLE and severe neurological impairment classifiable as demyelinating syndrome (DS) according to 1999 ACR nomenclature and case definition for NPSLE $^{1}$ and according to the NPSLE attribution algorithm. ${ }^{3}$ She was treated with $500 \mathrm{mg}$ MPRE pulses for 3 consecutive days, followed by $500 \mathrm{mg}$ CYC one single pulse and RTX $1 \mathrm{~g}$, which was then repeated 14 days later according to previously described regimen. ${ }^{9}$ After the second dose of RTX, oral PRE $25 \mathrm{mg}$ /day, rapidly tapered to $7.5 \mathrm{mg}$ /day in 6 months, and mycophenolate mofetil (MMF) $2 \mathrm{~g} /$ day were prescribed as maintenance therapy, but she could tolerate only $1 \mathrm{~g} /$ day because of diarrheal symptoms. Since the beginning of treat-

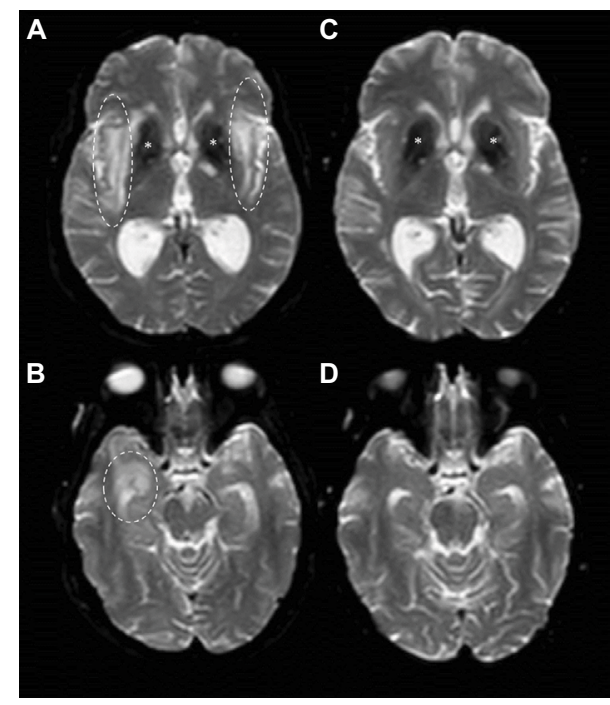

Figure $3 \mathrm{DWI}$ images before and after B-cell depleting therapy. Notes: Brain MRI DWI sequences from February 2016 (A and B) showed hyperintensity in right temporal lobe (dashed circles), external capsule and claustrum (dashed ovals) with increased apparent diffusion coefficient (not shown) suggesting vasogenic edema. *Basal ganglia calcification. On September 2016, 6 months after RTX treatment, brain MRI DWI sequences (C and D) showed complete disappearance of edema.

Abbreviations: MRI, magnetic resonance imaging; DWI, diffusion-weighted imaging; RTX, rituximab.

ment, she experienced progressive improvement in nystagmus, diplopia, dysarthria, cranial nerve palsies and multiple mononeuritis, which had completely disappeared at the 3-month follow-up visit. Ataxia disappeared within 6 months, while cognitive dysfunction, albeit greatly improved, showed incomplete resolution after 1 year of follow-up. Follow-up MRI revealed remarkable reduction in number and size of WM lesions (Figures 2 and 3).

\section{Discussion}

B cells carry out central roles in the pathogenesis of SLE through a combination of antibody-mediated and antibodyindependent actions. ${ }^{10-13}$ Despite conflicting evidence from clinical trials, BCDT was proven to be effective in several lupus manifestations by inhibiting the interaction between functionally activated B cells and $\mathrm{T}$ cells and also possibly by reducing the production of certain cytokines and complement activation, which could lead to rapid improvement in NPSLE manifestations. ${ }^{14-16}$ Emerging evidence highlighted BCDT as an effective and steroid-sparing treatment for renal and nonrenal lupus manifestations when associated with immunosuppressive maintenance. ${ }^{9,10}$ Nevertheless, RTX is still an off-label drug reserved to those SLE patients who have failed to respond to conventional immunosuppression.

A recent literature review showed that DS-SLE is burden by a great risk of death (up to $25 \%$ ) and disability (35-65\%), 
even in those cases treated with high-dose corticosteroids alone or in combination with CYC as induction therapy, because of unresponsiveness to drugs and high relapsing rate. ${ }^{7}$ In the reported case, we applied the principles of the two-hit treatment strategy recommended by EULAR using MPRE and RTX as induction therapies (first hit) and MMF during maintenance (second hit). It would be difficult to ascribe the response to one agent more than the others, but definitely this strategy was successful to lower inflammation, prevent accrue of damage, avoid new relapse and reduce the use of corticosteroids. This is worthy of mention especially given the propensity of corticosteroids for inducing damage in patients with SLE, especially in those with central nervous system involvement, and for precipitating new NPSLE events. ${ }^{17-19}$ In conclusion, RTX represents an effective therapeutic option for severe NPSLE and especially for DS-SLE providing to associate it with a conventional immunosuppressant as maintenance treatment to reduce the risk of flare and reduce corticosteroids dose.

\section{Disclosure}

The authors report no conflicts of interest in this work.

\section{References}

1. [No authors listed]. The American College of Rheumatology nomenclature and case definitions for neuropsychiatric lupus syndromes. Arthritis Rheum. 1999;42(4):599-608.

2. Piga M, Mathieu A. Managing CNS involvement in systemic lupus erythematosus. Int J Clin Rheumatol. 2011;6:547-567.

3. Bortoluzzi A, Scirè CA, Bombardieri S, et al. Development and validation of a new algorithm for attribution of neuropsychiatric events in systemic lupus erythematosus. Rheumatology (Oxford). 2015;54(5):891-898.

4. Piga M, Casula L, Perra D, et al. Population-based analysis of hospitalizations in a West-European region revealed major changes in hospital utilization for patients with systemic lupus erythematosus over the period 2001-2012. Lupus. 2016;25:28-37.

5. Hanly JG, Urowitz MB, Su L, et al. Short-term outcome of neuropsychiatric events in systemic lupus erythematosus upon enrollment into an international inception cohort study. Arthritis Rheum. 2008;59(5):721-729.
6. Zirkzee EJ, Huizinga TW, Bollen EL, et al. Mortality in neuropsychiatric systemic lupus erythematosus (NPSLE). Lupus. 2014;23:31-38.

7. Piga M, Chessa E, Peltz MT, Floris A, Mathieu A, Cauli A. Demyelinating syndrome in SLE encompasses different subtypes: do we need new classification criteria? Pooled results from systematic literature review and monocentric cohort analysis. Autoimmun Rev. 2017;16(3): 244-252.

8. Bertsias GK, Ioannidis JP, Aringer M, et al. EULAR recommendations for the management of systemic lupus erythematosus with neuropsychiatric manifestations: report of a task force of the EULAR standing committee for clinical affairs. Ann Rheum Dis. 2010;69(12): 2074-2082.

9. Ezeonyeji AN, Isenberg DA. Early treatment with rituximab in newly diagnosed systemic lupus erythematosus patients: a steroid-sparing regimen. Rheumatology (Oxford). 2012;51(3):476-481.

10. Pepper R, Griffith M, Kirwan C, et al. Rituximab is an effective treatment for lupus nephritis and allows a reduction in maintenance steroids. Nephrol Dial Transplant. 2009;24(12):3717-3723.

11. Steri M, Orrù V, Idda ML, et al. Overexpression of the cytokine BAFF and autoimmunity risk. $N$ Engl J Med. 2017;376(17):1615-1626.

12. Wahren-Herlenius $M$, Dörner T. Immunopathogenic mechanisms of systemic autoimmune disease. Lancet. 2013;382(9894): 819-831.

13. Floris A, Piga M, Cauli A, Mathieu A. Predictors of flares in systemic lupus erythematosus: preventive therapeutic intervention based on serial anti-dsDNA antibodies assessment. Analysis of a monocentric cohort and literature review. Autoimmun Rev. 2016;15(7):656-663.

14. Piga M, Gabba A, Cauli A, Garau P, Vacca A, Mathieu A. Rituximab treatment for 'rhupus syndrome': clinical and power-Doppler ultrasonographic monitoring of response. A longitudinal pilot study. Lupus. 2013;22(6):624-628.

15. Narváez J, Ríos-Rodriguez V, de la Fuente D, et al. Rituximab therapy in refractory neuropsychiatric lupus: current clinical evidence. Semin Arthritis Rheum. 2011;41:364-372.

16. Tokunaga M, Saito K, Kawabata D, et al. Efficacy of rituximab (antiCD20) for refractory systemic lupus erythematosus involving the central nervous system. Ann Rheum Dis. 2007;66(4):470-475.

17. Gladman DD, Urowitz B, Rahman P, Ibañez D, Tam LS. Accrual of organ damage over time in patients with systemic lupus erythematosus. J Rheumatol. 2003;30(9):1955-1959.

18. Piga M, Peltz MT, Montaldo C, et al. Twenty-year brain magnetic resonance imaging follow-up study in systemic lupus erythematosus: factors associated with accrual of damage and central nervous system involvement. Autoimmun Rev. 2015;14(6):510-516.

19. Govoni M, Bombardieri S, Bortoluzzi A, et al. Factors and comorbidities associated with first central nervous system neuropsychiatric event in systemic lupus erythematosus: does a risk profile exist? A large multicenter retrospective cross sectional study on 959 Italian patients. Rheumatology (Oxford). 2012;51:157-168.
Open Access Rheumatology: Research and Reviews

\section{Publish your work in this journal}

Open Access Rheumatology: Research and Reviews is an international, peerreviewed, open access journal publishing original research, reports, editorials, reviews and commentaries on all aspects of clinical and experimental rheumatology in the clinic and laboratory including the following topics: Pathology, pathophysiology of rheumatological diseases; Investigation, treatment and

\section{Dovepress}

management of rheumatological diseases; Clinical trials and novel pharmacologi$\mathrm{cal}$ approaches for the treatment of rheumatological disorders. The manuscript management system is completely online and includes a very quick and fair peer-review system, which is all easy to use. Visit http://www.dovepress.com/ testimonials.php to read real quotes from published authors. 